\title{
The form of timing distributions in pigeons under penalties for responding early
}

\author{
KEN CHENG \\ University of Toronto, Toronto, Ontario, Canada
}

\begin{abstract}
The peak procedure was used in two experiments. Pigeons in the penalty group in Experiment 1 were rewarded with food in the first phase for the first peck after $12.5 \mathrm{sec}$ had elapsed since the onset of a keylight. In the second phase, reward was withheld if the pigeons pecked within $6.25 \mathrm{sec}$ after keylight onset. Responses in time were tabulated on occasional unrewarded tests in which the keylight was left on for $37.5 \mathrm{sec}$. Under the penalty contingencies, the response distribution in time remained nearly symmetric about the peak, while the spread of the distribution narrowed, and the time of peak responding came slightly earlier. The yoke group underwent a schedule of rewards similar to that for the penalty group, but without the penalty contingencies. Their response distributions remained similar throughout. The results of Experiment 1 were replicated in Experiment 2, which showed further that the changes due to the penalty contingencies did not generalize to the use of another key on which the penalty contingencies were not in effect. The narrower spread under the penalty contingencies is explained in terms of a change in threshold for when to start responding, and more weight being given to timing versus responding in the presence of the signal per se. No explanation was found for the change in the time of peak responding.
\end{abstract}

Recent studies on the timing of short intervals in rats and pigeons suggest the operation of an internal clock in timing (Cheng \& W. A. Roberts, 1989, 1991; Gibbon \& Church, 1984, 1990; S. Roberts, 1981, 1982; W. A. Roberts, Cheng, \& Cohen, 1989). The most influential theory of timing, scalar expectancy theory (Gibbon \& Church, 1984, 1990), suggests the operation of a single clock and a single memory system that generates behavior on the basis of the clock reading.

One paradigm often used to study timing is the peak procedure. Here, the animal is trained on a discrete-trials fixed interval (FI) schedule with a signal. A signal comes on at the start of a trial. After a fixed period of time has elapsed, the first target response, typically a barpress or a keypeck, is rewarded. Occasionally, the signal is left on for a far longer time than usual, and reward is withheld. The distribution of responses in time on these empty trials constitutes the data.

The distributions obtained from empty trials in the peak procedure reveal three properties. (1) They are nearly symmetric about the time of peak responding, which occurs at some interval after the signal onset that is close to the FI. A small positive skew, however, is typically found (e.g., S. Roberts, 1981; W. A. Roberts et al., 1989). (2) The standard deviation of the distribution or

This research was supported by a National Sciences and Engineering Research Council grant to K. Cheng. I thank Sara Shettleworth for kindly letting us use her equipment, Richard Westwood for help in conducting the experiments, and two anonymous reviewers for helpful comments. Correspondence about this article should be sent to Ken Cheng, Department of Psychology, University of Toronto, Toronto, ON, Canada M5S 1A1. the spread (the width of the distribution at a fixed proportion of the peak rate of responding) is a constant proportion of the FI, a property consistent with Weber's law (Cheng, in press; Cheng \& W. A. Roberts, 1991; Gibbon \& Church, 1990). (3) While the response distribution averaged across many trials has an approximately normal shape, the pattern of responding on individual trials is better characterized as a break-run-break pattern. Gibbon and Church (1990) reported that the animal starts the trial at a roughly constant low rate of responding, then switches abruptly to a high rate of responding, and later reverts back to a low rate of responding. Unpublished results from my laboratory confirm this pattern. The response rate during the run phase in both sets of data seems to show a small rise followed by a drop. Thus, the Gaussian shape of the averaged distribution arises mainly from variations across trials in when the run starts and ends.

Briefly, scalar expectancy theory accounts for performance on the peak procedure as follows. At the signal onset at the start of a trial, the clock is started after some delay. A criterion time for the expected time of reward is retrieved from reference memory. A decision process compares the elapsed time on the clock with the criterion time. When the ratio of elapsed time to criterion time is greater than a threshold, the animal starts the run phase. The same ratio is compared with another threshold to determine when to end the run. This comparison uses, perhaps, a different sample criterion time from reference memory.

The comparison of a ratio of elapsed time to criterion time will generate performance consistent with Weber's law. The roughly symmetric shape of the averaged distribution means that the thresholds for when to start and 
end the run are, on the average, roughly equidistant from the criterion time. The small positive skew in the averaged distribution can come from variance in either clock speed or criterion time. Variation in either source produces larger variance in when the run ends than in when the run starts, because each source of variance produces variance in proportion to the amount of time elapsed. A moderate positive correlation across trials between when the run starts and when it ends (Gibbon \& Church, 1990) also implies variance in either or both of these sources, and possibly in the delay to start the clock as well. A faster clock speed, a shorter delay to start the clock, or an earlier criterion time translates to an earlier start and an earlier end; a slower clock speed, a longer delay to start the clock, or a later criterion time means a later start and a later end. In this case, if the positive correlation is due in part to variance in criterion time, the same criterion time must have been used for both comparisons (when to start and when to end the run). Independent criterion times for the two comparisons would produce no correlation between the start and the end of the run.

A moderate negative correlation between the start of the run and the length of the run phase suggests threshold variance (Gibbon \& Church, 1990). A threshold for the start of the run closer to the criterion time produces a later start of the run and a shorter run, whereas a threshold farther from the criterion time produces an earlier start and a longer run. This is the case whether the second threshold (for the end of the run) on the trial is independent or linked in some fashion to the first threshold. Thus, threshold variance across trials is implied, but it is unclear whether the two thresholds on a trial are independent.

In the experiments reported here, pigeons were tested in the peak procedure to see how immutable the psychophysical property of a roughly symmetric shape in the average response distribution is. After some standard training in the peak procedure, the birds were trained in a condition designed to produce skewed distributions. They were penalized for responding too early after the onset of the signal. Pecking within the first half of the FI extinguished the signal and deprived the subject of reward for that trial. No penalties were imposed for late pecking.

Three plausible solutions to these contingencies can be stated. The first hypothesis is that the shape of the distribution and the spread of the distribution in the standard training are immutable. This can come about because the distributions of thresholds used are immutable. To solve the early penalty contingencies, the birds must set a later criterion time and peak at a later time. This hypothesis predicts a symmetric distribution under the early penalty contingencies, with a later time of peak responding and a similar spread. The second hypothesis is that the birds can learn to sharpen the average distribution on the rise side, and thus produce an asymmetric distribution with the time of peak responding about the same as before. This can come about if the birds move the threshold distribution for when to start the run closer to the criterion time while leaving the threshold distribution for when to end the run unaltered. The third hypothesis is that the birds sharpen the average distribution on both sides of the time of peak responding, producing a symmetric distribution that is narrower than before, but with a similar time of peak responding. This can come about because the thresholds for when to start and end the run phase are both moved closer to the criterion time. This must happen if the two thresholds used on a trial are linked, and the animals shift the threshold to avoid the penalty for early pecking.

\section{EXPERIMENT 1}

In Experiment 1, two groups of pigeons, a penalty group and a yoke group, were run with the peak procedure, using an FI of $12.5 \mathrm{sec}$. After the birds in the penalty group had stabilized on the standard task, they were gradually trained to withhold responding in the first $6.25 \mathrm{sec}$ of the signal; responding too early meant obtaining no reward for that trial. After behavior had stabilized again, the penalty for responding early was lifted. In a final phase, the reward schedule during the penalty phase was reinstated, but this time, reward was not contingent upon behavior. Instead, the birds were yoked to their earlier behavior; they were rewarded only on trials on which they obtained reward during the penalty phase. The yoke group was run after the birds in the penalty group were finished. Each bird's schedule of trials was yoked to the performance of 1 bird in the penalty group. When the birds in the penalty group obtained a reward, so did its counterpart in the yoke group. When the bird in the penalty group was penalized, the trial for its counterpart was ended shortly after signal onset without reward. Thus, the yoke group received the same frequency of rewards as did the penalty group, on a similar schedule. Whether reward was obtainable on a trial, however, was not contingent on behavior.

\section{Method}

\section{Subjects}

Six naive adult White King pigeons (Columba livia) were used as subjects. The birds were kept between $80 \%$ and $85 \%$ of freefeeding weight. They had constant access to water and grit. Three birds were assigned to the penalty group and 3 to the yoke group.

\section{Apparatus}

Three operant chambers measuring approximately $31 \mathrm{~cm}$ in each dimension were used. The single keylight used in the experiment was either at the center or on the left side of the panel. The food hopper was directly below the key. The reward throughout consisted of three 20-mg pigeon pellets delivered into the hopper, which was lit during the 3.7-sec reward period and dark otherwise.

\section{Procedure for the Penalty Group}

The birds in the penalty group were trained and tested in four phases. Throughout experimentation, four types of trials were given. On a training trial, a green keylight came on to signal the start of the trial. The first keypeck after $12.5 \mathrm{sec}$ turned off the keylight and led to reward. The intertrial interval in darkness was $10 \mathrm{sec}$ throughout experimentation. On a test trial, the green keylight came on. It was turned off 37.5 sec later, irrespective of how the bird 
behaved. No reward was dispensed. On a penalty trial, the green keylight came on. Responding the first $2.5,3.75,5$, or $6.25 \mathrm{sec}$ (depending on the penalty period, as described below) extinguished the keylight, and no reward was dispensed. Otherwise, the first peck after $12.5 \mathrm{sec}$ led to reward. On an unrewarded trial, the green keylight came on. It was turned off at the midpoint of one of the first five $1.25-\mathrm{sec}$ intervals, independently of behavior. These trials were used as yoked trials to mimic the unrewarded penalty trials while the contingencies of the penalty were removed.

Phase 1: Control. Baseline data were gathered in Phase 1, which consisted of training and test trials. The birds were first given 8 sessions consisting solely of training trials. The first 4 sessions consisted of 50 trials, and the next 4 sessions, of 100 trials. Twenty sessions then followed in which test trials were mixed with training trials. The first 2 sessions consisted of 80 training trials and 20 test trials; the last 18 sessions consisted of 100 training trials and 25 test trials. The last 10 sessions were analyzed.

One order of trials was used throughout. It was chosen at random with the following constraints: (1) each block of 10 trials contained exactly two test trials; (2) two test trials were separated by at least two training trials; and (3) the first four trials were training trials.

Phase 2: Penalty contingencies. In this phase, the birds were trained gradually to withhold pecking during the first $6.25 \mathrm{sec}$ of the signal. Test data were gathered after they had learned the task. At the beginning, responding within the period of $2.5 \mathrm{sec}$ from the start of the keylight was punished by nonreward. Thereafter, the penalty period was increased by $1.25 \mathrm{sec}$ when a bird obtained $75 \%$ or more of the rewards on two consecutive sessions. After 20 sessions of less than $75 \%$ success at one particular penalty period, training was terminated, and the bird was tested thereafter at the previous penalty period. After two consecutive successes $(75 \%$ or better) at the 6.25 -sec penalty period, a bird was given three more training sessions before testing began. One bird (11) failed the 6.25 sec penalty period after 28 sessions of training in total, and was tested thereafter with a 5-sec penalty period. The other 2 birds passed the 6.25 -sec penalty period in 25 and 28 sessions of training in total. The first 6 training sessions had 100 trials, and the next 8 sessions, 120 trials; thereafter, the birds were given 140 trials per session.

Test trials were then mixed with the penalty trials. In the first 3 sessions, 20 test trials were mixed with 110 penalty trials. Thereafter, 25 test trials were mixed with 130 penalty trials. The first 30 trials of each session were penalty trials. Thereafter, the order of trials used in Phase 1 was used, except that penalty trials replaced training trials. Each bird was given 38 test sessions in total, with the last 28 analyzed.

Phase 3: Control. In this phase, the birds were retrained and retested with the penalty contingencies lifted. For 8 sessions, they were given 200 training trials per session. Thereafter, they were given 20 sessions, with 130 training trials mixed with 25 test trials. The order of trials was the same as that in Phase 2, except that training trials replaced penalty trials. The last 10 sessions were analyzed.

Phase 4: Yoke. In this phase, the birds were yoked to their performance in the penalty phase (2). A trial that was rewarded in Phase 2 was also rewarded in this phase (i.e., a training trial was given). A trial that was penalized in Phase 2 was terminated unrewarded (i.e., an unrewarded trial was given). If the bird had pecked in the $n$th 1.25 -sec bin after the keylight onset in the penalty phase, the corresponding trial in this phase terminated at the middle of the $n$th 1.25-sec bin, irrespective of how the bird behaved. Test trials were given in the same ordinal positions. In all, 20 sessions were given, yoked to Sessions 11-30 in Phase 2. The last 10 sessions were analyzed.

A few irregularities due to error and equipment failure arose. On four occasions (none were used for data analysis), a bird was placed in a chamber other than its usual. On the first session in
Phase 2 containing test trials, Birds 11 and 4 were given four fewer penalty trials and one fewer test trial by a programming error. On one session in the training phase of Phase 2, Bird 4 was given a 3.75-sec penalty period instead of the intended 5-sec period. Session 10 in the test phase of Phase 2 was aborted for Bird 11, because of equipment failure. An extra session was given before Session 10 was repeated.

\section{Procedure for the Yoke Group}

The birds in the yoke group were yoked to the 3 birds in the penalty group, being matched approximately by weight. They were run after experimentation with the penalty group was through. Each bird was run in the operant chamber in which its yoke counterpart had been run. The experiment took place in four phases, each yoked to the corresponding phase in the penalty group. Phases 1,3 , and 4 followed the procedures used on the penalty group identically. Only Phase 2, which was the penalty phase for the penalty group, differed. In the training sessions of Phase 2 , the exact sequence of rewarded and unrewarded trials had not been recorded for the penalty group. Yoking to an exact sequence of rewards and nonrewards was thus not possible. Instead, birds in the yoke group were presented the same total number of rewards per session as were their yoke counterparts. In each session, the sequence of rewarded and nonrewarded trials was chosen at random. A rewarded trial was an FI 12.5-sec training trial. A nonrewarded trial consisted of the green keylight's being illuminated and extinguished 1.25, $2.5,3.75$, or $5 \mathrm{sec}$ later, independently of the bird's behavior. The duration of illumination was $1.25 \mathrm{sec}$ less than the penalty period of the yoke counterpart during the corresponding session. During the test sessions of Phase 2, the exact sequence of rewards and nonrewards for the penalty group was recorded, and the birds in the yoke group were exposed to identical trial sequences. For each subject and each session, a successful trial on the part of the yoke counterpart meant a rewarded FI 12.5-sec training trial, while a penalized trial on the part of the yoke counterpart meant an unrewarded trial. When a yoke counterpart was penalized for pecking in the $n$th 1.25-sec period from the keylight onset, the keylight was extinguished in the middle of the $n$th 1.25 -sec period for the yoked subject. In each phase, the same sessions that were analyzed for the penalty group were also analyzed for the yoke group.

A few irregularities occurred. Bird $\mathbf{4 0}$ was given the wrong number of rewarded trials on two occasions in the training phase of Phase 2. In one session in Phase 2, the keylight malfunctioned for Bird 33 on an undetermined number of trials. Bird 39 was placed in a chamber other than its usual one, on two occasions. Data from Session 11 of the last phase were lost for Bird 40; data from Session 10 were substituted for analysis.

\section{Data Analysis}

For all subjects in each phase of the experiment, the first 10 sessions with test trials were not analyzed, in order to ensure that behavior on tests had stabilized. In the data from Phases 2 and 4 , only test trials on which the previous trial was rewarded were analyzed. Unrewarded trials affect the peak time and the shape of the distribution on the following trial, at least in rats (S. Roberts, 1981). On the test trials analyzed in each phase, the numbers of pecks in 1.25 -sec intervals since keylight onset were tabulated. Mean normalized response rates in each of the 301.25 -sec bins were plotted. In addition, parameters of peak time, peak rate, and width at $50 \%$ peak rate (spread) were calculated. For peak time, an initial median was calculated on the basis of response rates in 1.25-sec bins. A second median was calculated on a restricted range of the distribution. This range ran from the end nearest the median to the point on the other side of the median that put the first median at the center of the range. For example, if the first median calculation yielded a value of $15 \mathrm{sec}$, the next calculation would be done over the range 0-30 sec even if responses occurred after $30 \mathrm{sec}$ during test trials. 
The process was iterated until two iterations produced medians differing by less than $0.0625 \mathrm{sec}$. The last median was taken to be the peak time. Peak rate was the rate of responding at the peak time and was calculated by linear interpolation between the two bins on either side of the peak time. Width at $50 \%$ peak rate was the width of the response distribution at $50 \%$ of the peak rate. The points on either side of the peak that were at $50 \%$ of the peak rate were found by linear interpolation, and the duration between those points was the spread parameter. Spread is an estimate of how wide the response distribution is relative to its height, because it measures width relative to a constant proportion of the peak rate (height). Justification for the use of these calculation procedures has been given elsewhere (Cheng, 1989; S. Roberts, 1981).

\section{Results and Discussion}

\section{Penalty Group}

Results for the two groups of birds are discussed separately in turn. Response distributions in all phases for the penalty group are presented in Figure 1. The distributions for the second control and yoke phases ( 3 and 4 ) were similar and have been combined. All distributions have a similar, nearly symmetric shape, with a rise toward the end of the test trial. The penalty phase, however, produced a narrower distribution that peaked earlier than the first baseline phase (Control 1). After the penalty contingencies were lifted, the spread of the distribution stayed similar, while peak time shifted to the right once more. Individual data on spread and peak time presented in Figures 2 and 3 confirm these impressions. Each bird individually showed the same pattern.

A within-subject analysis of variance (ANOVA) was performed on spread (Figure 2), peak time (Figure 3), and peak rate (not presented). Spread differed significantly across phases $[F(3,6)=142.3, p<.01]$. Post hoc Scheffé tests showed that the Control 1 phase (Phase 1) differed from the other three conditions at the .01 level. The last three phases did not differ from each other. Peak time also differed significantly across phases $[F(3,6)=$ $9.54, p<.05$ ]. Post hoc Scheffé tests showed that the penalty phase (Phase 2) had a lower peak time than did the second control phase (Phase 3 ) and the yoke phase (Phase 4). The difference between the first baseline phase (Phase 1) and the penalty phase (Phase 2) approached significance $(p<.10)$. No other paired comparisons between conditions approached significance. Peak rate showed no significant differences across phases.

The symmetry of the distribution in the penalty phase was examined more closely. The distribution from each bird was normalized; the distributions were shifted left/right so that the bins containing the peak times lined up; the three distributions were averaged and then renormalized. The results, along with those of the same analysis for the first control phase, are shown in Figure 4. They show near symmetry about the peak time in the penalty phase.

\section{Yoke Group}

The response distribution in each phase for the yoke group (not presented) was again nearly symmetric about the peak. But contrary to the pattern for the penalty group, the parameters of peak time and spread were similar in all phases. Figures 5 and 6 present these parameters for individual cases; a within-subject ANOVA revealed no significant differences across conditions in either parameter. Peak rate also showed no significant variation across phases.

The performance of the yoke group shows that the differences in peak time and spread found in the penalty group were attributable to the penalty contingencies, and

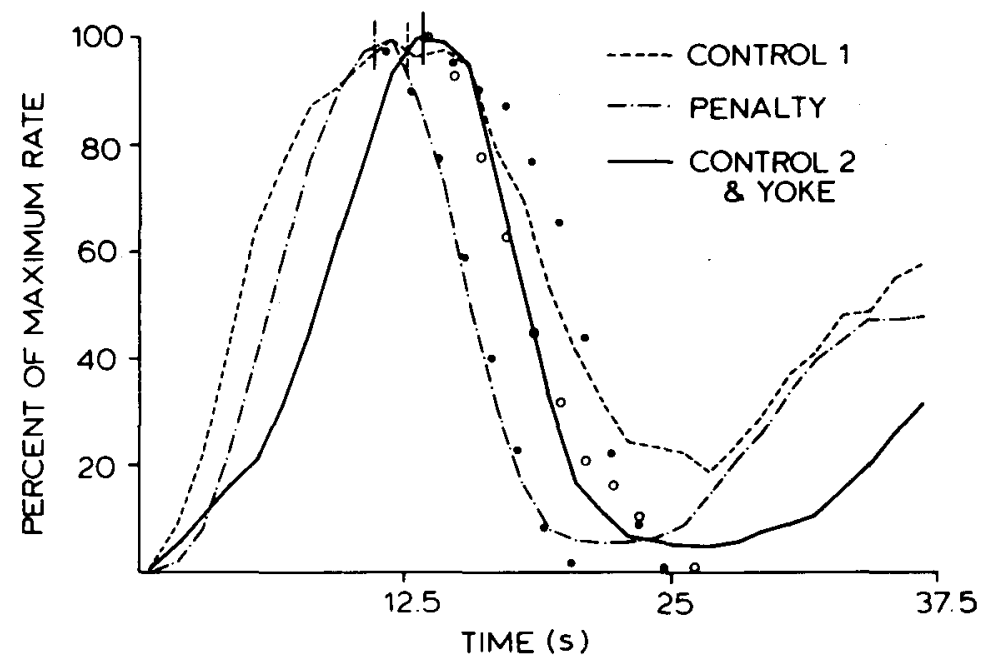

Figure 1. Normalized response distributions in the different phases of Experiment 1 for the penalty group. (The distributions were normalized as a percentage of the maximal bin for each bird, and were then averaged across birds and renormalized as a percentage of the maximal bin. The vertical dashes show the peak times of the averaged distributions. The open and filled dots show the data points to the left of the peak time reflected about the peak time.) 


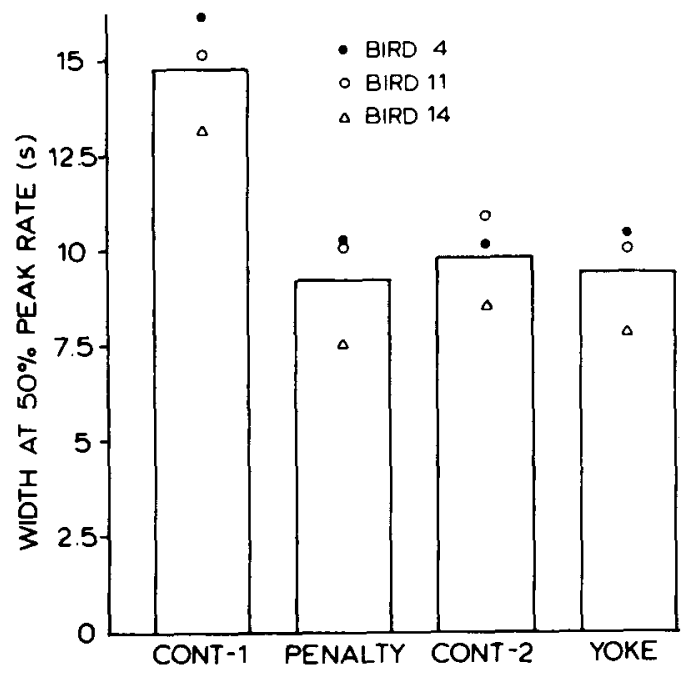

Figure 2. Width of the response distribution at $50 \%$ of peak rate in the different phases of Experiment 1 for the penalty group. (The symbols show individual data points, and the bar shows the average across birds.)

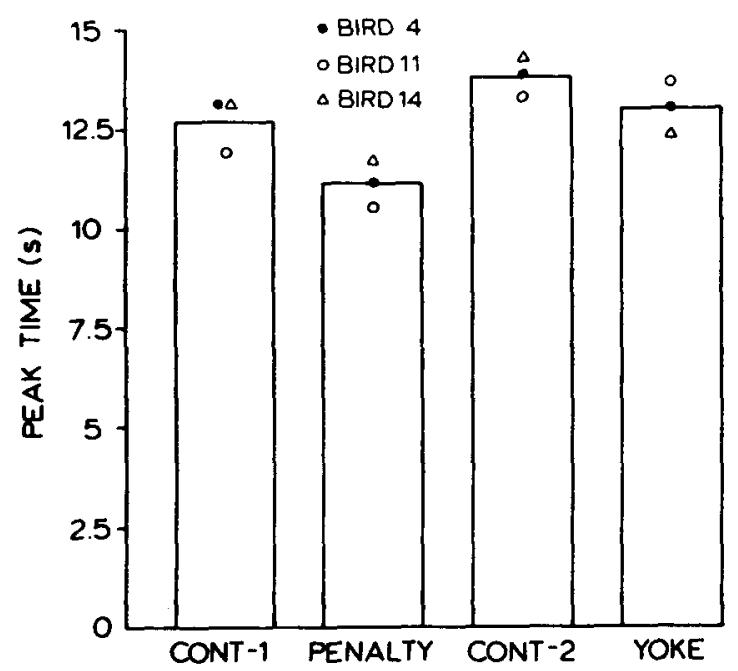

Figure 3. Time of peak responding in the different phases of Experiment 1 for the penalty group. (The symbols show individual data points, and the bar shows the average across birds.)

not to additional training or the reduced frequency of rewards in the penalty phase. The yoke birds underwent a similar amount of training and a similar schedule of reward as the penalty birds. In sum, when the penalty for responding early was imposed, the birds showed a nearly symmetric response distribution with a narrower spread, and they peaked earlier. After the penalty contingencies were removed, peak time came later again, but spread stayed narrow. Peak rate showed no significant changes under the penalty contingencies.

That peak times should be earlier in the penalty phase is counterintuitive. Penalty for responding early ought to encourage a later rather than an earlier peak time. A penalty in the first few seconds amounts to an $S$ - on one side of the generalization gradient. In other perceptual dimensions, an S- on one side of an S+ often leads to peak shift away from $S+$ in the direction away from $S-$, in what is commonly known as the peak shift phenomenon (Hanson, 1959). The results on peak times here are opposite to what would be predicted on the basis of the peak shift phenomenon.

\section{EXPERIMENT 2}

Experiment 2 was an attempt to replicate the pattern of results found in Experiment 1. It was also a test of whether the changes in peak time and spread as a result of the penalty contingencies would generalize to a different signal trained on the same FI 12.5-sec schedule for which the penalty contingencies were never in effect. Birds were trained on an FI 12.5-sec schedule on two keylights differing in color and location. A penalty for early responding was then imposed for one keylight, while the other was not presented. After exposure to the penalty contingencies, both signals were presented, separately, one with the penalty contingencies, and one without. Both immediate and long-term generalization to the unpenalized signal were examined. In a final phase, the penalty contingencies were lifted.
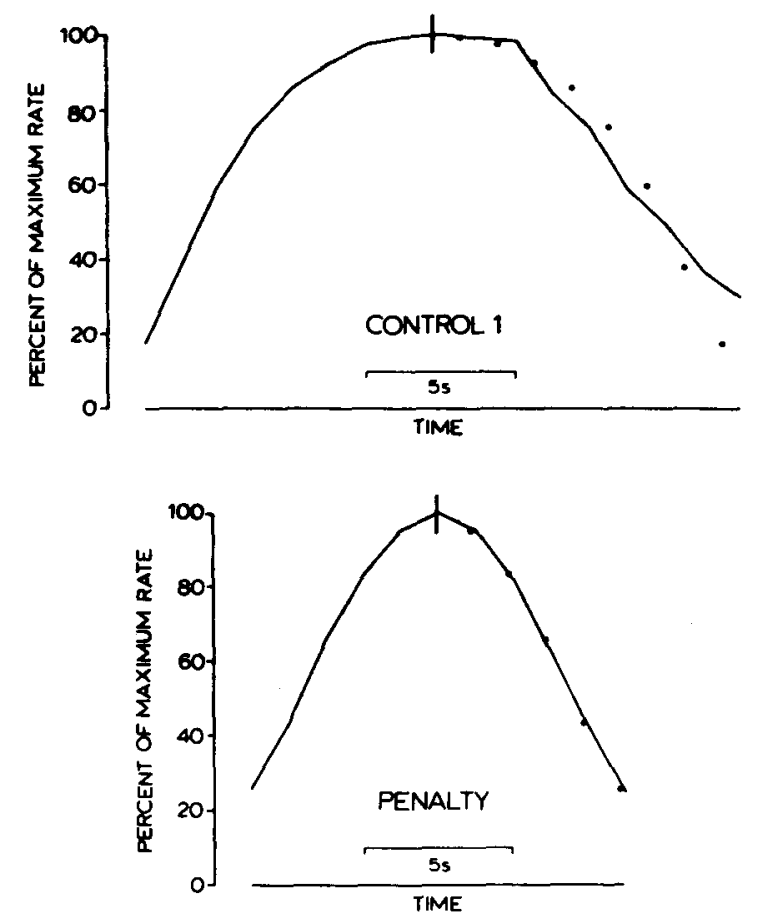

Figure 4. Normalized response distributions in the first control phase and penalty phase of Experiment 1 for the penalty group. (The distributions were normalized as a percentage of the maximal bin for each bird. The bins in which the peak time was found for each bird were then lined up, and the individual distributions were averaged across birds and renormalized as a percentage of the maximal bin. The vertical dashes show the peak times of the averaged distributions. The dots show the data points to the left of the peak time reflected about the peak time.) 


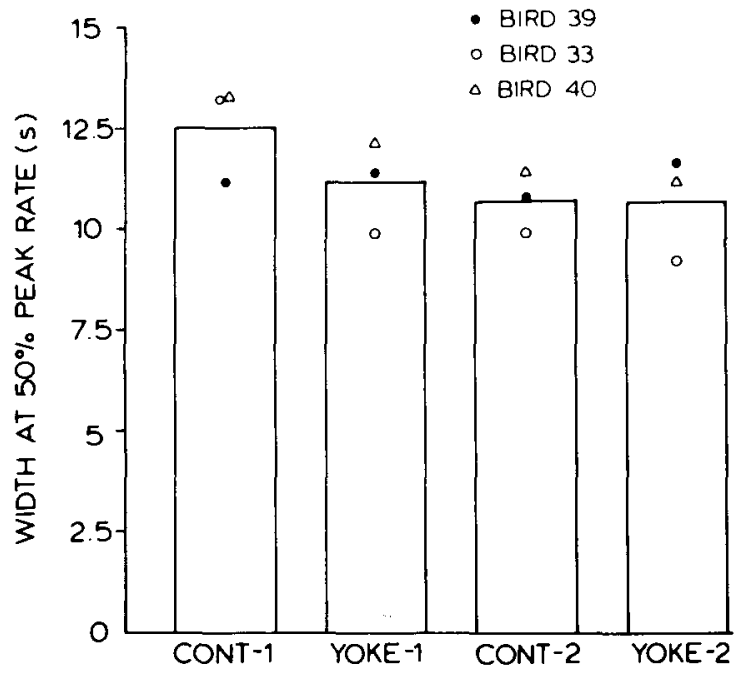

Figure 5. Width of the response distribution at $50 \%$ of peak rate in the different phases of Experiment 1 for the yoke group. (The symbols show individual data points, and the bar shows the average across birds.)

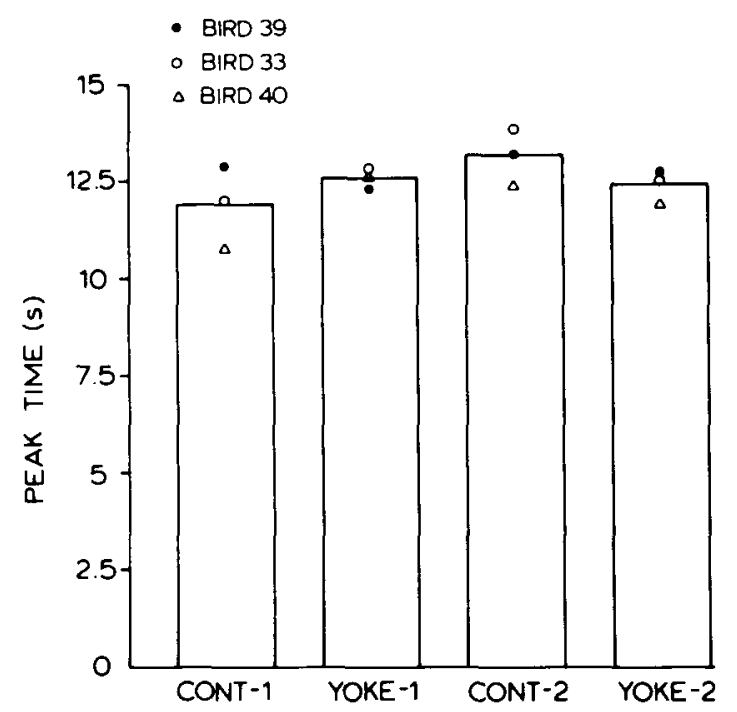

Figure 6. Time of peak responding in the different phases of Experiment 1 for the yoke group. (The symbols show individual data points, and the bar shows the average across birds.)

\section{Method}

Subjects

Three White King pigeons kept under the same conditions as those in Experiment 1 were the subjects. These birds were experienced in the peak procedure, but they had not faced the penalty contingencies imposed in the experiment. Just before the experiment, they were in fact being trained and tested on an FI 12.5-sec schedule.

\section{Apparatus}

The birds were tested in three of the four operant chambers used in Experiment 1. In addition to the keylight above the feeder, which was lit green, a keylight to the right, lit white, was used.

\section{Procedure}

Phase 1: Control. In each session in this and in all subsequent phases, only one keylight was presented to the birds. In this phase, each bird was given 18 sessions with the green keylight and 18 sessions with the white keylight, in strict alternation across days. Bird 42 was given an extra session with the green keylight to replace lost data. Sessions 6-18 were used for data.

Each session consisted of 120 trials, with 100 training trials and 20 test trials. The first 20 trials were training trials. Thereafter, each block of 10 trials contained 2 test trials. Test trials were separated by at least 2 training trials. One randomly chosen order was used throughout, for both keylights.

A training trial was programmed with an FI 12.5-sec contingency. The first keypeck after $12.5 \mathrm{sec}$ since the onset of keylight terminated the keylight and was rewarded. A test trial consisted of a 75-sec presentation of the keylight, which was then terminated without reward. Data collection and analysis were as in Experiment 1.

Phase 2: Penalty contingencies. In this phase, the birds were first trained on the green keylight with penalties for responding early, during which time sessions with the white keylight were not presented. The training procedure followed that in Experiment 1 up to the criterion of a 6.25 -sec penalty period. Bird 41 was trained for 22 sessions past the criterion (46 sessions in total); Bird 42 was trained for 23 sessions past the criterion ( 44 sessions total); Bird 43 failed to pass the criterion for a 5-sec penalty period after 30 sessions ( 49 sessions in total). It was tested subsequently with a 5 -sec penalty period. One session with Bird $\mathbf{4 2}$ was aborted due to equipment failure.

At this point, test trials were included, and sessions with the white keylight (without penalty contingencies) alternated with sessions with the green keylight (penalty contingency still in effect). The order of trials used in Phase 1 continued to be used. The birds were given 25 sessions with each keylight, the last 20 of which were used for data.

Phase 3: Control. In this phase, the conditions of Phase 1 were reinstated. Thus, the green keylight no longer carried the penalty contingencies. Each bird was given 19 sessions with each keylight, the last 14 of which were analyzed.

\section{Results and Discussion}

The response distributions once again narrowed during the penalty phase for the green key, for which the penalty contingency was in effect (Figure 7). For the second control phase for the green key, the spread of the response distributions was intermediate between the other two phases. For the white key, for which the penalty contingency was never in effect, the spread remained similar throughout (Figure 7). Spread did not narrow during the second phase, either in the first two test sessions or at asymptote. The peak times were again slightly but consistently earlier during the penalty phase for the green key (Figure 8). For the white key, the peak times were similar in all phases (Figure 8). Thus, no immediate or longterm generalization to the white, unpenalized, key was apparent. The shape of the response distribution during the penalty phase for the green key was once again nearly symmetric (Figure 9).

Two-way within-subject ANOVAs were performed on peak rates, spreads, and peak times, with phase of experiment and response key as factors. The asymptotic data from the second phase of the white key were used. Spreads differed across phases $[F(2,4)=12.99, p<.05]$, and 


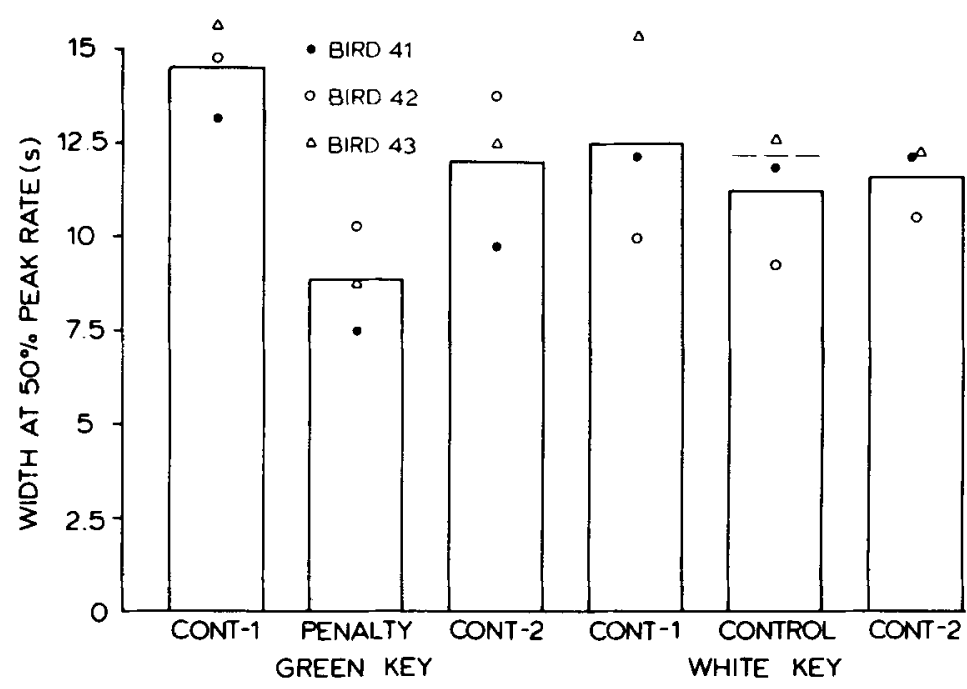

Figure 7. Width of the response distribution at $50 \%$ of peak rate in the different phases of Experiment 2 for each response key. (In the second phase of the experiment, the green key had the penalty contingencies, and the white key did not. The symbols show individual data points, and the bar shows the average across birds. The dashed line shows the average for the first two test sessions with the white key. The response distribution on these two sessions for 1 bird was truncated after 30 bins out of 60 because it showed an exceptionally high rate of responding at the end of the tests.)

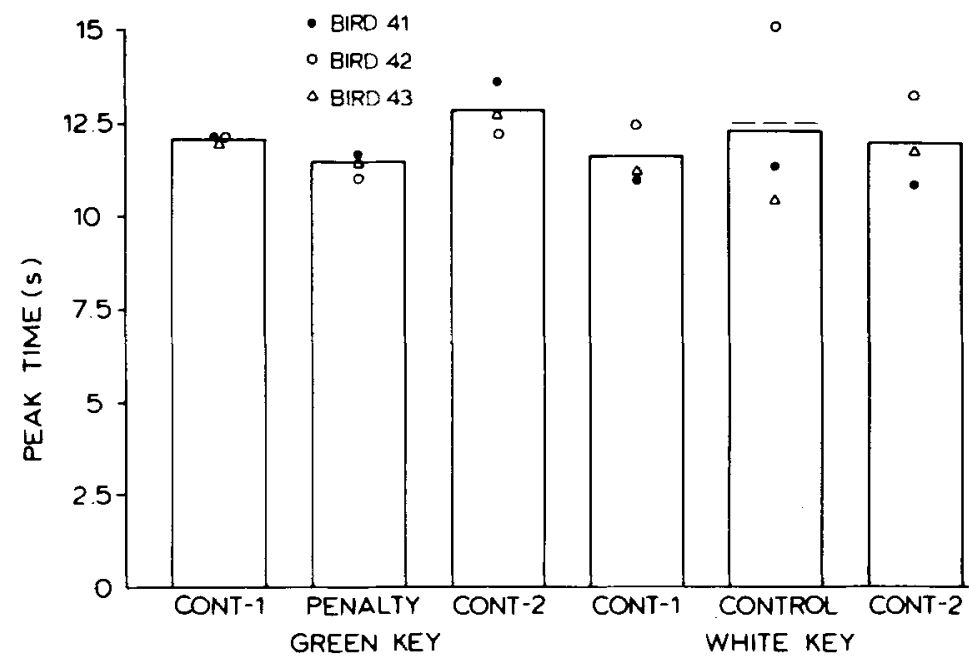

Figure 8. Time of peak responding in the different phases of Experiment 2 for each response key. (In the second phase of the experiment, the green key had the penalty contingencies, and the white key did not. The symbols show individual data points, and the bar shows the average acroes birds. The dached line shows the average for the first two test sessions with the white key. The response distribution on these two sessions for 1 bird was truncated after 30 bins out of 60 because it showed an exceptionally high rate of responding at the end of the tests.) 

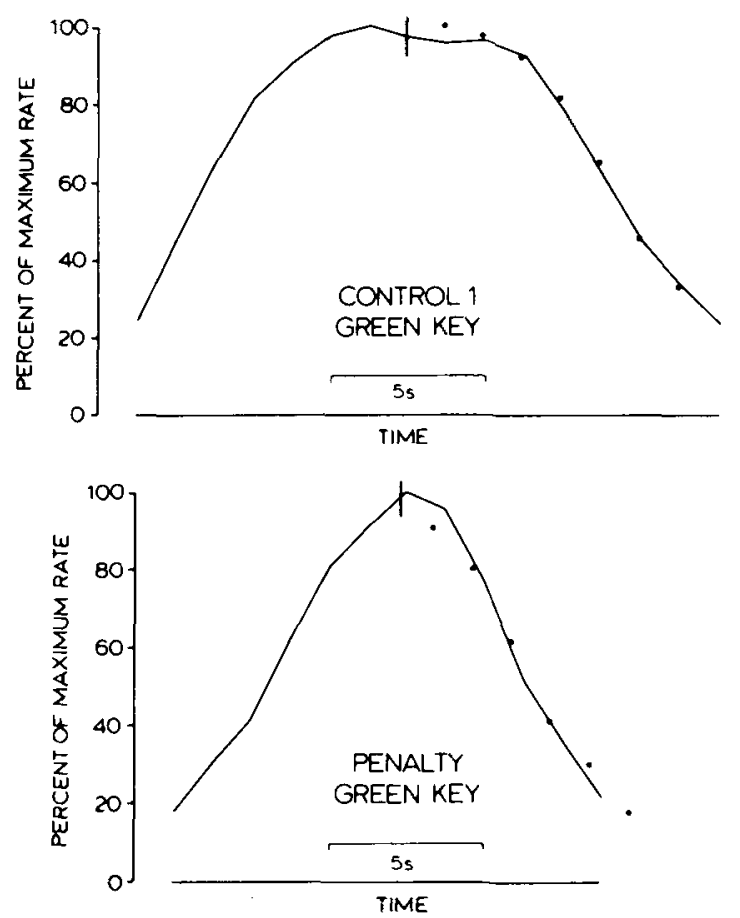

Figure 9. Normalized response distributions on the green key in he first control phase and penalty phase of Experiment 2. (The disributions were normalized as a percentage of the maximal bin for sach bird. The bins in which the peak time was found for each bird were then lined up, and the individual distributions were averaged ccross birds and renormalized as a percentage of the maximal bin. The vertical dashes show the peak times of the averaged distribuions. The dots show the data points to the left of the peak time reflected about the peak time.)

nore importantly, this main effect was qualified by a reponse key $\times$ phase interaction $[F(2,4)=17.74, p<$ 05]. For peak times and peak rates, no significant efects were found.

For both spreads and peak times, it appears from FigIres 7 and 8 that all the differences were found with the ;reen key. A one-way within-subject ANOVA on each rariable was conducted for the phases of the green key lone. For spreads, the phases differed at the .01 level Jf significance $[F(2,4)=34.97]$. Post hoc Scheffé tests evealed that each phase differed from each of the other hases at the .05 level of significance. Peak times for the yreen key also differed across phases $[F(2,4)=12.83$, $,<.05]$. Post hoc Scheffé tests revealed that the two :ontrol phases did not differ at the .05 level of signifiance, but that the penalty phase differed from the averige of the two control phases at the .05 level of signifiance. Thus, peak times were once again earlier during he penalty phase. Similar one-way ANOVAs performed or the white key revealed no differences across phases or either spread or peak time, and the analysis did not 'hange when the first two sessions of the second phase vere substituted for the asymptotic data.

For the green key, the symmetry of response distribuions for the first control phase and the penalty phase was examined more closely. The normalization procedure used in Experiment 1 was again performed, and the results (Figure 9) showed symmetric distributions in both phases.

In sum, for the green key, for which penalty contingencies were in effect during the penalty phase, the results replicated in the main what was found in Experiment 1 . Spread was narrower during the penalty phase, and peak time was slightly earlier. For the second control phase, peak times came once again slightly later, and spreads also widened somewhat. This last result differs from Experiment 1, in which the spread for the penalty group stayed narrow through the control and yoke phases after the penalty phase. For the white key, for which penalty contingencies were never in effect, spread and peak time remained similar throughout. Thus, the narrowing of the response distribution in the peak procedure as a function of penalties for responding early did not generalize to apply to the use of a different key for which the penalty was not in effect.

\section{GENERAL DISCUSSION}

The results of Experiment 1 showed that when pigeons on the peak procedure were penalized for pecking early, their response distributions in time narrowed while remaining nearly symmetric about the peak. They also peaked at a slightly earlier time. When the penalty contingencies were lifted, peak times shifted back to original levels, while the distributions remained narrow and symmetric. This pattern of results is attributable to the penalty contingencies. Another group that underwent a similar reward schedule without the penalty contingencies showed no significant changes in peak time or spread. The results of Experiment 2 replicated those of Experiment 1 , with but one exception. In the control phase after the penalty contingencies, response distributions once again widened, although not to the extent of the first control phase. It also showed that the distributions on another key for which the penalty contingencies were never in effect remained similar throughout. This suggests that the narrowing of the distributions as a function of penalty for early responding does not generalize to apply to the use of a nonpenalized key.

Thus, the attempt to induce a nonsymmetric response distribution failed. Pigeons responded to the penalty for early pecking with a sharper generalization gradient on both sides of the peak time. Under conditions designed to elicit asymmetric distributions, the distributions remained nearly symmetric about the peak.

Why did the pigeons narrow their response distributions under the penalty contingencies? Two complementary explanations can be given. One is that the birds shifted their threshold for starting the run phase closer to the criterion time. Another is that the birds reduced responding not related to the timing task. Making the threshold for starting the run phase stricter provides a natural explanation for how the pigeons coped with the penalty contingencies. If the distribution for thresholds to start the run did shift to the right, however, the distribution of thresh- 
olds for when to end the run must have shifted to the left in order for the average distribution to remain nearly symmetric under the penalty contingencies. This arises naturally if the thresholds on a trial are always linked (e.g., always equidistant from the criterion time), which in effect reduces two threshold parameters to one. If the two thresholds are independent, some ad hoc explanation will have to be given for why the threshold distribution for when to end the run should shift as well under the penalty contingencies.

S. Roberts (1981) argued that for rats, responses in the peak procedure are attributable to two sources, one related to timing the FI, and one not related (see also Eberling \& S. Roberts, 1989; S. Roberts, in press). On the basis of timing, the animal responds with a sharply peaked distribution. But the animal also associates rewards with making the response in the presence of the signal per se, irrespective of time. On this nontemporal basis, responding is assumed to be fairly constant throughout the interval. The particular spread in the observed distribution depends on the proportions of these two strategies used; the more the animal bases its responses on timing, the narrower the distribution. Eberling and S. Roberts (1989) found that throughout training on the peak procedure, the spread of the distribution and the tail rate-the asymptotic rate of responding at the end of the distribution-always covaried. With practice, spread narrowed and tail rate decreased, in the same proportion over sessions. Striatal lesions led to a wider spread and a correspondingly higher tail rate. They suggested that the spread and the tail rate both reflect the amount of responding based on timing. Striatal lesions made the rats put less weight on timing, and more on responding in the presence of the signal per se.

A similar interpretation can be given for the present results. The penalty contingencies forced the pigeons to put more weight on timing. After all, under the penalty contingencies, the presence of the green light provided no guarantee of reward. If more weight is put on timing, the spread of the response distribution would decrease without affecting other parameters such as the symmetry of the distribution. It must also be assumed that in some cases, even when the penalty contingencies were later lifted, the weight placed on timing did not change, since the distributions stayed narrow after the penalty was lifted in Experiment 1.

Thus, in one explanation, the birds eliminated some responses related to timing the signal, while in the other explanation, the birds eliminated some responses unrelated to timing. Most likely, both explanations apply. To be successful, the birds must withhold all pecks in the penalty period, both those stemming from timing and those stemming from nontemporal sources.
These interpretations explain the narrowing of the distributions with the penalty contingencies. They do not explain why peak time should shift earlier under the penalty conditions. Plausible reasons can be given for why the peak time might come later under the penalty contingencies for early responding. But I can give no good explanation for why peak time should arrive earlier.

In sum, the experiments presented here were attempts to induce an asymmetric response distribution in the peak procedure. The key manipulation consisted of penalizing the subjects for responding too early in the signaled interval. Response distributions stayed symmetric under these conditions while their spread narrowed. This was interpreted as arising from (1) a later threshold for when to start responding at a high rate, and (2) more weight given to timing as a basis for responding. The penalty contingencies also produced earlier peak responding, for which no satisfactory explanation was found.

\section{REFERENCES}

ChENG, K. (1989). The vector sum model of pigeon landmark use. Journal of Experimental Psychology: Animal Behavior Processes, 15, 366-375.

Cheng, K. (in press). Three psychophysical principles in the processing of spatial and temporal information. In W. K. Honig \& J. G. Fetterman (Eds.), Cognitive aspects of stimulus control. Hillsdale, NJ: Erlbaum.

Cheng, K., \& Roberts, W. A. (1989). Timing multimodal events in pigeons. Journal of the Experimental Analysis of Behavior, 52 , 363-376.

Cheng, K., \& Roberts, W. A. (1991). Three psychophysical principles of timing in pigeons. Learning \& Motivation, 22, 112-128.

Eberling, J. L., Roberts, S. (1989, November). Striatum lesions selectively change one measure of time discrimination. Paper presented at the meeting of the Psychonomic Society, Atlanta.

Gibbon, J., \& CHURCH, R. M. (1984). Sources of variance in an information processing theory of timing. In H. L. Roitblat, T. G. Bever \& H. S. Terrace (Eds.), Animal cognition (pp. 465-488). Hillsdale, NJ: Erlbaum.

Gibmon, J., Church, R. M. (1990). Representation of time. Cognition, 37, 23-54.

Hanson, H. M. (1959). Effects of discrimination training on stimulus generalization. Joumal of Experimental Psychology, 58, 321-333.

ROBERTS, S. (1981). Isolation of an internal clock. Joumal of Experimental Psychology: Animal Behavior Processes, 7, 242-268.

ROBERTS, S. (1982). Cross-modal use of an internal clock. Journal of Experimental Psychology: Animal Behavior Processes, 8, 2-22.

RoberTs, S. (in press). Use of independent and correlated measures to divide a time-discrimination mechanism into parts. In D. E. Meyer \& S. Kornblum (Eds.), Attention and performance XIV: A silver jubilee.

Roberts, W. A., Cheng, K., \& Cohen, J. S. (1989). Timing visual and auditory signals in pigeons. Joumal of Experimental Psychology: Animal Behavior Processes, 15, 23-35.

(Manuscript received January 22, 1991; revision accepted for publication October 5, 1991.) 